\title{
Information consumption by Reissner-Nordström black holes
}

\author{
Andrew Strominger* \\ Institute for Theoretical Physics, and Department of Physics, University of California, Santa Barbara, California 93106 \\ Sandip P. Trivedi ${ }^{\dagger}$ \\ Department of Physics, California Institute of Technology, Pasadena, California 91125
}

(Received 19 July 1993)

\begin{abstract}
The low-energy scattering of charged fermions by extremal magnetic Reissner-Nordström black holes is analyzed in the large- $N$ and $S$-wave approximations. It is shown that (in these approximations) information is carried into a causally inaccessible region of spacetime, and thereby effectively lost. It is also shown that there is an infinite degeneracy of quantum black hole ground states, or "remnants," which store, but will not reveal, the information. A notable feature of the analysis, not shared by recent analyses of dilatonic black holes, is that the key physical questions can be answered within the weak coupling domain. We regard these results as strong evidence that effective information loss occurs in our Universe.
\end{abstract}

PACS number(s): 04.60. $+\mathrm{n}$, 11.80.Fv, 97.60.Lf

\section{INTRODUCTION}

Extremal black holes provide a simple laboratory in which to study quantum-mechanical aspects of black holes. There are three general possibilities which have been discussed for the outcome of a scattering experiment in which a particle is sent into an extremal black hole and Hawking reemitted: (I) The scattering is unitary, with a finite number of quantum states for the black hole; (II) the scattering is unitary with an infinite number of nants"; (III) the scattering is not unitary, and information is destroyed.

Extensive analyses of extremal black holes in dilaton gravity at large $N$ over the last year [1] show no evidence that possibility (I) might be realized, while recent work [2] has shown that possibilities (II) and (III) are much less distinct than previously suspected.

One feature of the large- $N$ analysis of dilatonic black holes has been in some ways disappointing: Gravitational collapse inevitably leads to a singularity at which the large- $N$ approximation breaks down. Fortunately, some key physical questions are not affected by this breakdown. For example, possibility (I) can still be ruled out at large $N$. However, one cannot determine which of the possibilities (II) or (III) is realized without solving a strongly coupled quantum problem.

We were thus motivated to search for a model in which possibility (II) is demonstrably realized at weak coupling. ${ }^{1}$ After running around in several circles we realized that such a model was under our noses: real-world magnetic Reissner-Nordström black holes. Although the

\footnotetext{
*Electronic address: andy @ denali.physics.ucsb.edu

†Electronic address: trivedi@ theory3.caltech.edu

${ }^{1}$ See Ref. [3] for related efforts.
} asymptotic quantum states of the black hole, or "rem-

structure of their extremal ground state is much more complex than that of their dilatonic cousins, they have two big advantages: large $N$ can tame their dynamics, and they exist as solutions to the Einstein-Maxwell equations, without the introduction of unobserved fields such as a dilaton.

We wish to study long-wavelength scattering of $S$-wave charged fermions by an extremal Reissner-Nordström black hole with a large magnetic charge and radius, both given by $Q$ (we take $Q>0$ ). To render this problem tractable, we make the $S$-wave approximation in which all higher angular modes are suppressed. Naively, one expects that, at wavelengths large relative to $Q$, this approximation is good. However, unlike the dilatonic case studied in Ref. [1], there are several subtleties [4] which have so far prevented a careful justification of the approximation, and we cannot be sure that it is valid. ${ }^{2}$ For $N+1$ flavors of fermions, the effective two-dimensional $S$-wave

\footnotetext{
${ }^{2}$ One subtlety is that incoming long-wavelength modes may produce regions of high curvature either near the origin or the inner Cauchy horizon. We shall argue later that these regions are irrelevant to the issue of effective information loss. Another subtlety has to do with the fact that the centrifugal barrier seen by the higher partial waves turns off near the horizon. This means firstly that there are an infinite number of short-distance but low-energy modes near the horizon. We believe these should not be present in a long-distance effective theory, but we do not know how to define such a theory in a manner consistent with Lorentz invariance and energy conservation. It also means that there are long-distance low-energy higher angular momentum modes near the horizon which might be excited as quantum fluctuations. As was discussed in Ref. [5] the tidal forces seen by these modes, unlike the $S$-wave, blow up at the horizon, thus once excited they might have consequences which are unaccounted for in the $S$-wave approximation.
} 
theory is described by

$$
\begin{aligned}
S=\frac{1}{2 \pi} \int d^{2} \sigma \sqrt{-g} & {\left[e^{-2 \phi} R+2 e^{-2 \phi}(\nabla \phi)^{2}+2-2 Q^{2} e^{2 \phi}\right.} \\
& \left.-\frac{1}{2} \sum_{i=1}^{N}\left(\nabla f_{i}\right)^{2}\right] .
\end{aligned}
$$

The two-dimensional metric appearing in (1) is related to the four-dimensional metric by

$$
d s^{2}=g_{\alpha \beta} d \sigma^{\alpha} d \sigma^{\beta}+e^{-2 \phi} d^{2} \Omega,
$$

with $\alpha$ and $\beta$ ranging over $(r, t)$. The scalar field $\phi$ measures the (logarithm of) the area of two spheres of constant radius. The first four terms in (1) arise directly from dimensional reduction of the four-dimensional Einstein-Maxwell action. The last term arises from the bosonization of fermion $S$-wave modes studied by Callan and Rubakov [6] in grand unified theories (GUT). One charged liner combination of the original $N+1$ flavors acquires a mass from electromagnetic effects. The dynamics of this mode was studied for dilaton black holes in Ref. [7], but because of its mass it decouples at sufficiently low energies. The two-dimensional relic of the four-dimensional gauge field is suppressed in (1), as it cannot be excited by the neutral fields and so may be consistently neglected. Previous work on the model defined in (1) and related models obtained by dimensional reduction can be found in Refs. [5,8].

One might hope that for large $Q$ particle-hole scattering could be adequately analyzed in a semiclassical loop expansion of the reduced theory, but, in fact, large $N$ will be needed in addition to the $S$-wave approximation for several reasons. First, as pointed out in Ref. [9], the temperature fluctuations of a near-extremal charged black hole go as

$$
\frac{\Delta T}{T} \sim\left[\frac{\hbar}{\sqrt{M-Q}}\right)^{1 / 2},
$$

so the leading semiclassical formula for the temperature (and radiation rate) becomes unreliable very near extremality. But in the large- $N$ limit, where $\hbar \rightarrow 0$ (while $N \rightarrow \infty$ keeping $N \hbar$ fixed) we see that

$$
\frac{\Delta T}{T} \rightarrow 0
$$

so that near-extremal black holes are indeed characterized by a definite temperature.

A second problem with the loop expansion was discussed in Ref. [5]. For a nonextremal ReissnerNordström black hole, the one-loop contribution to the expectation value of the stress tensor diverges on the inner (but not the outer) horizon. This is related to the classical instability of the inner horizon, as studied in many papers [10]. This divergence persists, albeit in a softened form, in the extremal limit in which the two horizons coalesce. Since the one-loop corrections are divergent, the loop expansion is clearly unreliable.

Although frightening at first, these divergences are, in fact, rather benign and can be controlled within the $1 / N$ expansion. The leading large- $N$ equations, in which oneloop quantum back reaction is included, have solutions which are in a sense "near" to corresponding classical solutions. In particular [5], there is an extremal, zerotemperature ground-state solution with causal structure identical to that of the classical solution. The large- $N$ geometry is near to its classical counterpart, but third and higher derivatives of the fields are divergent near the horizon. We shall see in this paper that divergences encountered in particle-hole scattering are also benign, although the behavior of the stress tensor near the horizon leads to an unexpected nonanalytic large- $N$ mass-area relationship, which differs from the classical result even at large $Q$ sufficiently near extremality.

\section{CALCULATION}

Previous analyses of large- $N$ two-dimensional gravity have been largely carried out in conformal gauge. This gauge is somewhat awkward in the present context. For example, even the classical solutions are known only implicitly in this gauge. A more convenient choice is the light-cone gauge, ${ }^{3}$ for which the two-dimensional metric takes the form

$$
d s^{2}=-h(d v)^{2}+2 d r d v,
$$

$\sqrt{-g}=1$ and the scalar curvature is $R=-\partial_{r}^{2} h$. In this gauge one can solve the classical equations and obtain an analytic expression, known as the Vaidya metric, for arbitrary null infalling matter, characterized by a stress tensor obeying $T_{r a}=0$ :

$$
h=1-\frac{2 M(v)}{r}+\frac{Q^{2}}{r^{2}},
$$

where $2 \partial_{v} M=T_{v v}$. The large- $N$ trace and dilaton equations may then be written in the form

$$
\begin{aligned}
& \square \phi=\partial_{r} \Sigma=\Sigma \partial_{r} U+\frac{Q^{2} e^{4 \phi}\left(1+\gamma e^{2 \phi}\right)}{1-\gamma e^{2 \phi}}-\frac{e^{2 \phi}}{1-\gamma e^{2 \phi}}, \\
& R=-\partial_{r}^{2} h=\frac{2}{1-\gamma e^{2 \phi}}\left(e^{2 \phi}-2 Q^{2} e^{4 \phi}-\Sigma \partial_{r} \phi\right),
\end{aligned}
$$

where

$$
\begin{aligned}
& \Sigma \equiv 2 \partial_{v} \phi+h \partial_{r} \phi, \\
& U \equiv 2 \phi-\frac{1}{2} \ln \left(1-\gamma e^{2 \phi}\right),
\end{aligned}
$$

and

$$
\gamma=N \hbar / 24
$$

A future (past) apparent horizon is a zero of $\Sigma\left(\partial_{r} \phi\right)$, which implies $(\nabla \phi)^{2}=0$. One important linear combination of the constraint equations is local:

\footnotetext{
${ }^{3}$ Previous light-cone gauge analyses of dilatonic black holes can be found in Ref. [11].
} 


$$
\begin{aligned}
e^{2 \phi} l^{a} l^{b} T_{a b}^{Q} & \equiv \frac{1}{2} \gamma e^{2 \phi}\left[\frac{1}{2} h \partial_{r}^{2} h-\frac{1}{4}\left(\partial_{r} h\right)^{2}+\partial_{v} \partial_{r} h\right] \\
& =\partial_{v} \Sigma+\frac{h}{2} \partial_{r} \Sigma-\frac{1}{2} \Sigma^{2}-\frac{1}{2} \partial_{r} h \Sigma
\end{aligned}
$$

where the components of the null vector $l$ are $\left(l^{r}, l^{v}\right)=(h / 2,1)$. Fortunately, the other linear combination, which is nonlocal, shall not be needed.

The extremal solutions were studied in Ref. [5] and found to be of two kinds referred to therein as the even and odd extensions. Here we shall focus on the odd extension, which reduces to the classical solution as $\hbar \rightarrow 0$, and denote it as $\phi_{0}(r)$ and $^{4} h_{0}(r)$. This solution has a timelike singularity at the "origin" where $e^{-2 \phi_{0}}=\gamma$. Near the horizon $r_{H}$ it was shown [5] that the fields have the nonanalytic behavior

$$
\begin{aligned}
& \phi_{0}-\phi_{H}=\beta x|x|^{\delta}, \\
& h_{0}=\alpha_{1} x^{2}+\alpha_{2} x^{3}|x|^{\delta},
\end{aligned}
$$

where $\phi_{H} \equiv \phi\left(r_{H}\right), x \equiv r-r_{H}$, and

$$
\delta=\frac{3}{2}\left[\left[1+\frac{8 \gamma}{3\left(e^{-2 \phi_{H}}-\gamma\right)}\right]^{1 / 2}-1\right],
$$

with

$$
e^{-2 \phi_{H}}=Q^{2}\left(\frac{1+\sqrt{1+4 \gamma / Q^{2}}}{2}\right) .
$$

$\delta$ tends to zero for large $Q$. To leading order in $\gamma / Q^{2}$,

$$
\begin{aligned}
& \alpha_{1}=\frac{1}{Q^{2}}, \\
& \alpha_{2}=-\frac{2}{Q^{2}}, \\
& \beta=-\frac{1}{Q}, \\
& r_{H}=Q,
\end{aligned}
$$

and

$$
e^{-\phi_{H}}=Q
$$

While for large $Q$ one can safely use these approximations to $\alpha_{1}, \alpha_{2}, \beta, r_{H}$, and $\phi_{H}$, we do not omit terms subleading in $1 / Q$ in the expression for $\delta$ because such an approximation would break down very near the horizon ${ }^{5}$ [at $x$ less than of order $Q \exp \left(-Q^{2} / \gamma\right)$ ]. The nonanalyticity in (10) leads to divergences for example in the second derivative of the curvature.

Let us now consider an incoming matter shock wave ${ }^{6}$

\footnotetext{
${ }^{4}$ The even solutions are also interesting since they correspond to spacetimes free from any malevolent singularities. But their stability and response to perturbations cannot be studied in the approximations used here.

${ }^{5}$ Note that there is an issue of orders of limits here: we take $N \rightarrow \infty$ before $\mu \rightarrow 0$.

${ }^{6}$ Strictly speaking shock waves are not allowed in the longdistance effective field theory, but the case of a smooth pulse is qualitatively similar.
}

along $v_{0}$ whose classical stress tensor obeys

$$
l^{a} l^{b} T_{a b}^{f}=2 \mu \delta\left(v-v_{0}\right) .
$$

We wish to compute, following Ref. [12], $\phi$ and $h$ perturbatively in $\mu$ in a Taylor expansion above the shock wave. $\phi$ is continuous across the shock wave, while $h$ has a discontinuity which is determined by the constraints and is classically equal to $-2 \mu / r$. $\Sigma$ [defined in (7)] also has a discontinuity $\delta \Sigma$ across $v_{0}$, which, according to (5), obeys

$$
\partial_{r}\left(e^{-U} \delta \Sigma\right)=0
$$

The integration constant is determined from the asymptotic boundary condition $\delta \Sigma \rightarrow 2 \mu / r^{2}$. One thereby obtains

$$
\delta \Sigma=2 \mu e^{U} .
$$

Near the horizon $r_{H}, \Sigma_{0}$ (the value of $\Sigma$ below the shock wave) has a higher-order zero:

$$
\Sigma_{0} \approx \alpha_{1} \beta(1+\delta) x^{2}|x|^{\delta} \text {. }
$$

Since $\delta \Sigma$ is nonzero at $r_{H}$, this zero is split into two simple zeros which are (by definition) the inner and outer apparent horizons, as illustrated in Fig. 1. To leading order in $\gamma / Q^{2}$ and $\mu / Q$ one finds that the locations of the horizons are given by

$$
r_{ \pm} \approx r_{H} \pm Q\left(\frac{2 \mu}{Q}\right)^{1 /(2+\delta)}
$$

Comparing with (10) and recalling that the dilaton measures the area of the two spheres, we see that the area $A_{H}$

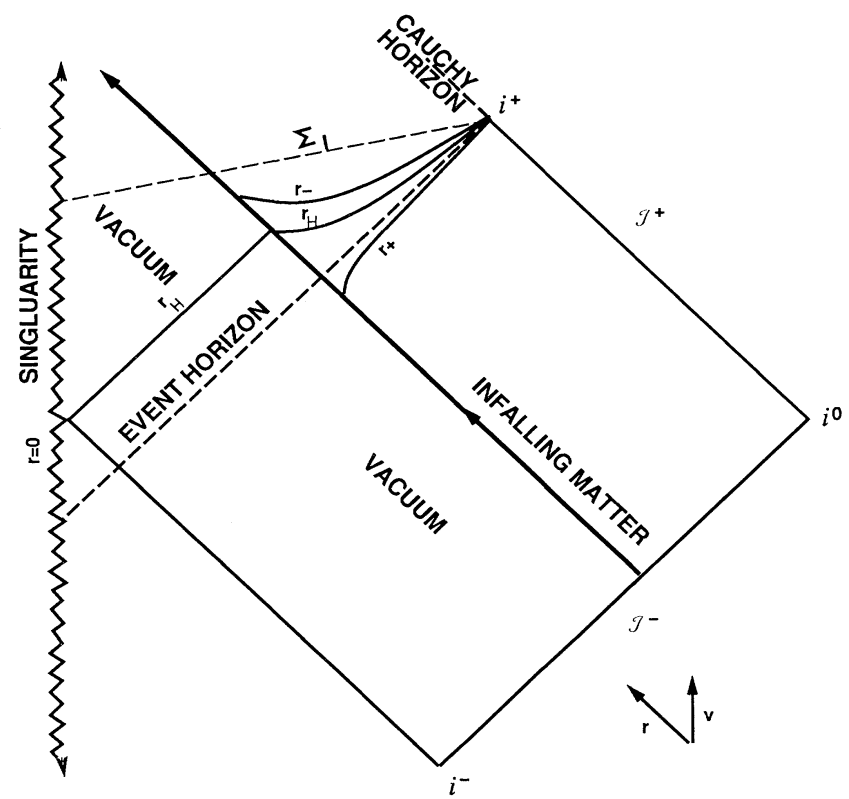

FIG. 1. A shock wave incident on an extremal ReissnerNordström black hole splits the apparent horizon $r_{H}$ into a pair of apparent horizons $r_{ \pm}$, which then exponentially decays back to $r_{H}$. The event horizon is outside $r_{H}$. The asymptotic spacelike surface $\Sigma$ is positioned so that the shock wave intersects it at large radius and weak coupling, and it avoids the Cauchy horizon. 
of the outer horizon obeys

$$
A_{H}-A_{0} \simeq 8 \pi Q^{2}\left[\frac{2 \mu}{Q}\right)^{(1+\delta) /(2+\delta)},
$$

where $A_{0}$ is the extremal area. Thus the mass-area relation is nonanalytic. Notice that no matter how small $\delta$ is, there is always some value of $\mu\left(\mu \sim Q e^{-1 / \delta}\right)$ below which (23) is not well approximated by the classical relation $A_{H}-A_{0} \sim 8 \pi \sqrt{2 \mu Q^{3}}$.

The mass of the black hole will, of course, decrease due to Hawking radiation and we expect it to settle back to extremality. To study this, we first calculate the trajectories of the two apparent horizons, denoted by $\hat{r}_{ \pm}$. We again work to leading order in $\gamma / Q^{2}$ and $\mu / Q$. Since $\Sigma$ vanishes along $\hat{r}_{ \pm}$one has

$$
\partial_{v} \Sigma=-\partial_{r} \Sigma \partial_{v} \widehat{r}_{ \pm} \text {. }
$$

Now (20) and (21) imply that

$$
\partial_{r} \Sigma\left(r_{ \pm}\right) \simeq \mp \frac{2}{Q^{2}}\left[\frac{2 \mu}{Q}\right]^{(1+\delta) /(2+\delta)} .
$$

Similarly (9) implies that, at $r_{ \pm}$,

$$
\begin{aligned}
\frac{h}{2} \partial_{r} \Sigma+\partial_{v} \Sigma=\frac{\gamma e^{2 \phi}}{2}( & \frac{h_{0}}{2} \partial_{r}^{2} \delta h+\frac{\delta h}{2} \partial_{r}^{2} h_{0}-\frac{1}{2} \partial_{r} h_{0} \partial_{r} \delta h \\
& \left.+\partial_{v} \partial_{r} \delta h\right]+e^{2 \phi} l^{a} l^{b} T_{0 a b}^{Q}
\end{aligned}
$$

It turns out that the dominant contribution for small $\mu$ is given by the second term on the right-hand side, which involves $\delta h$ with no derivatives. To evaluate this term we need to know $\delta h$ just above the shock wave which, according to (6) obeys

$$
\partial_{r}^{2} \delta h=\frac{2 \partial_{r} \phi \delta \Sigma}{1-\gamma e^{2 \phi}}
$$

so that

$$
\delta h=4 \mu \int d r \frac{1}{\gamma}\left(-1+\frac{1}{\sqrt{1-\gamma e^{2 \phi}}}\right) .
$$

(The integration constants are fixed by the requirement that $\delta h$ asymptotically vanish.) Furthermore, to leading order, $h \partial_{r} \Sigma$ vanishes so that (26) reduces to

$$
\partial_{v} \Sigma\left(r_{ \pm}\right) \simeq-\gamma \mu / Q^{5} \text {. }
$$

Equations (24) and (22) then imply that, just above the shock wave,

$$
\partial_{v} \hat{r}_{ \pm} \simeq-\frac{\gamma\left(\widehat{r}_{ \pm}-r_{H}\right)}{4 Q^{2}} .
$$

To proceed further, we evoke the adiabatic approximation in which the black hole is taken to evolve slowly so that its dynamic geometry may be approximated by a sequence of static ones. We expect the adiabatic approximation to be good for large $Q^{2}$, but have been unable to carefully justify this. In this approximation (30) continues to hold everywhere along $\hat{r}_{ \pm}$. Thus the inner horizon moves out towards $r_{H}$ while the outer horizon moves in towards $r_{H}$, and the black hole exponentially approaches its extremal ground state. Note, however, that while the black hole is excited, the trajectories of $\phi=\phi_{H}$ and $r=r_{H}$ are spacelike. The event horizon is therefore shifted outward (relative to the original apparent horizon) by the scattering process, as illustrated in Fig. 1.

Also, within the adiabatic approximation (22) relates the position of the outer horizon $r_{+}$to $\mu$. Equation (30) then implies that

$$
\partial_{v} \mu \simeq-\frac{\gamma}{2 Q^{3}} \mu
$$

so that the mass decays exponentially back to its extremal value as

$$
\mu(v) \simeq \mu e^{-\gamma\left(v-v_{0}\right) / 2 Q^{3}} .
$$

There are two regions in which the large- $N$ equations used here cannot be trusted. The first is near the origin $e^{2 \phi}=\gamma$ (denoted by $r=0$ in Fig. 1), where the curvature becomes large and higher-dimension corrections to the Einstein-Maxwell theory are important. The second is the future Cauchy horizon, or the extension of $\mathcal{J}^{+}$inside the event horizon. A timelike observer inside the black hole crosses this Cauchy horizon in finite time, yet is able to see all of the Universe outside the black hole before doing so. There is therefore a large energy concentration near this surface, the effects of which are subtle and have been analyzed in many papers [10].

Fortunately, physics outside the horizon is insensitive to the (intractable) behavior of the system in these regions. To see this, consider a Hamiltonian $H$ which evolves along the series of spacelike slices asymptotic to the slice $\Sigma=\Sigma_{T} \cup \mathcal{J}^{+}$where, as depicted in Fig. 1, $\Sigma_{T}$ is a spacelike surface inside the horizon. These slices can be chosen to completely cover the spacetime outside the horizon. $\Sigma_{T}$ can be chosen so that it avoids the difficult region near the future Cauchy horizon, and so that the intersection of the shock wave with $\Sigma_{T}$ is in weak coupling. Although $\Sigma_{T}$ extends into the strong-coupling region near $e^{2 \phi}=\gamma$, this does not present any difficulties because the system is unexcited in that region. The nontrivial dynamics are everywhere weakly coupled for all time, and our approximations should be valid.

\section{DISCUSSION}

We now argue that our results imply that an arbitrarily large amount of information can be sent into the black hole, and will never reemerge into the Universe from which it was thrown in. The black hole relaxes to extremality with a characteristic time

$$
t_{c}=Q^{3} / \gamma \text {. }
$$

Consider experiments in which an arbitrarily large number of wave packets are sent in from $\mathfrak{J}^{-}$spaced at intervals of $t_{c}$ seconds. In the process, an arbitrarily large amount of information is sent in. In order for no information loss to occur, in the asymptotic future, all correlations between the state inside and the state outside the horizon should be destroyed. This can occur only if the 
state inside is unique and independent of the initial state of the infalling matter. The preceding analysis shows that nothing catastrophic happens to the infalling matter as it crosses the apparent horizon so in the asymptotic future the state inside the event horizon (on $\Sigma_{T}$ ) will depend heavily on the incoming scattering state. Indeed, since the system is still weakly coupled on $\Sigma_{T}$, the quantum state of the left-moving conformal $f$ matter will be essentially the same as on $\mathscr{J}^{-}$. Thus in the course of this experiment an arbitrarily large amount of information will be carried into the causally inaccessible region inside the event horizon and will thereby be effectively lost.

It is also evident that, with respect to the time slicing described above, this is a theory with an infinite number of remnants. What we mean by this statement is that there are an infinite number of solutions of the large- $N$ constraint equations on a spacelike slice which are identical outside the horizon, but have differing $f$-matter configurations inside the horizon, corresponding to an infinite degeneracy of large- $N$ semiclassical quantum states. $^{7}$

However, it is important to note that the interpretation that the information is stored in these remnants may be dependent on the slicing. For example, we might have chosen the asymptotic interior surface $\Sigma_{T}$ so that the $f$ wave arrives at the singularity before intersecting $\Sigma_{T}$. In this case it is a logical possibility that the information is destroyed when it arrives at the singularity, in which case one would not conclude that the information is stored inside the black hole. ${ }^{8}$

Of course, physics outside the event horizon cannot, by causality, depend on the choice of slicing inside the event horizon, which we are therefore free to choose for our own convenience. Consequently, the observer outside the

\footnotetext{
${ }^{7}$ Actually, if we enforce the constraint that the incoming matter excitations are long wavelength on $\mathfrak{I}^{-}$, in accord with our approximations, this would not be the case because the incoming matter excitations must be late enough so that they are still in weak coupling when they arrive at $\Sigma$, yet early enough to avoid a potential pile up of energy density near the future Cauchy horizon. There are only a finite number of states in this finite interval above any given wavelength. This problem can be avoided by choosing a different surface $\Sigma_{H}$ defined as the (spacelike or null) surface along which $\phi$ takes the constant value $\phi_{H}$ characterizing the horizon of an unperturbed extremal solution. This is a geodesically complete surface which is everywhere in weak coupling. Since any finite point on $\Sigma_{H}$ is an infinite distance from $i^{+}$, there is clearly no pile up of energy density. Furthermore, because the event horizon is moved out by each scattering process, this surface is well behind the event horizon if many $f$ particles (and much information) are thrown into the black hole. The potentially infinite amount of information on $\Sigma_{H}$ is therefore unavailable to an observer on $\mathfrak{I}^{+}$.

${ }^{8}$ Another possibility is that boundary conditions might be specified at the timelike singularity to reflect the matter, and the information, up to the future Cauchy horizon and possibly on to the next universe. In this case all the information will be present on $\Sigma_{T}$ no matter how it is chosen.
}

horizon cannot possibly distinguish between actual information loss and storage by remnants. The choice of slicing made in this paper was motivated by the desire to avoid the difficult dynamics near the singularity, and it is consistent with this choice to describe the theory as having an infinite number of remnants.

We should note that although the calculations above were carried out in the $N \rightarrow \infty$ limit all the important conclusions continue to hold when $N$ (or $Q$ ) is sufficiently large but finite. The distinction is important to make because when $N \rightarrow \infty, \hbar \rightarrow 0$, so that the BekensteinHawking entropy of the black hole, which goes as $1 / \hbar$, goes to $\infty$. Thus it might be claimed that our conclusions are simply a consequence of working in a limit where the ground state is infinitely degenerate, and that at finite $N$ there would be an upper limit on the information the black hole can carry and an finite number of remnants. Finite $N$ differs from $N \rightarrow \infty$ in that we have to keep track of the quantum fluctuations in the metric and dilaton, and these might be potentially large close to the horizon. But the larger $N$ is, the closer one must approach the horizon in order for these effects to be significant. Similarly, the adiabatic approximation would break down for finite $N$ sufficiently close to extremality. But again for large enough $N$ this occurs only very near extremality. Thus by sending in energy at a judicious rate, for $N$ large but finite, one could keep the black hole close enough to extremality ( $\mu / Q$ small enough) for our approximations to hold, but far enough from extremality for the finite $N$ effects to be insignificant at the apparent horizons. The black hole would then respond according to the calculations above except for the first moments after it departs from extremality and the last moments before finally settling down. We would thus conclude that even for finite $N$ (when the entropy is finite) that the black hole can consume an arbitrarily large amount of information and store it in an infinite number of remnant states.

Finally, one may be concerned that this infinite degeneracy of states will lead to divergent black-hole pair production rates. In fact, magnetic black-hole pair production was computed semiclassically in Ref. [13] and found to be finite. The reason for this was discussed at length in Ref. [2]: extremal black holes do not behave quantum mechanically like elementary particles.

Thus we have found a system which can be seen, without resorting to speculations about strong-coupling dynamics, to solve the information puzzle by storing it in an infinite degeneracy of black-hole quantum states. Further this two-dimensional system might be a good approximation to real-world long-wavelength fermionmagnetic black-hole scattering.

\section{ACKNOWLEDGMENTS}

We wish to thank T. Banks, J. Bekenstein, S. Giddings, J. Hartle, G. Horowitz, M. O'Loughlin, J. Preskill, and L. Thorlacius for useful discussions. This work was supported in part by DOE Grant No. DEAC-03-8ER4050, and by the National Science Foundation under Grant No. PHY-89-04035. 
[1] C. G. Callan, S. B. Giddings, J. A. Harvey, and A. Strominger, Phys. Rev. D 45, R1005 (1992); for recent reviews see J. A. Harvey and A. Strominger, in Proceedings of the 1992 TASI Summer School in Boulder, Colorado (unpublished); S. B. Giddings, in String Quantum Gravity and Physics at the Planck Energy Scale, Proceedings of the Workshop, Erice, Italy, 1992, edited by N. Sanchez (unpublished).

[2] T. Banks, M. O'Loughlin, and A. Strominger, Phys. Rev. D 47, 4476 (1993).

[3] T. Banks and M. O'Loughlin, Phys. Rev. D 48, 698 (1993).

[4] T. Jacobson, Phys. Rev. D 44, 173 (1991); 48, 728 (1993).

[5] S. P. Trivedi, Phys. Rev. D 47, 4233 (1993).

[6] C. G. Callan, Phys. Rev. D 25, 2141 (1982); 26, 2058 (1982); Nucl. Phys. B212, 391 (1983); V. Rubakov, Pis'ma Zh. Eksp. Teor. Fiz. 33, 658 (1981) [JETP Lett. 33, 644 (1981)]; Nucl. Phys. B203, 311 (1982).

[7] M. Alford and A. Strominger, Phys. Rev. Lett. 69, 563 (1992).

[8] M. McGuigan, C. R. Nappi, and S. A. Yost, Nucl. Phys. B375, 421 (1992); O. Lechtenfeld and C. R. Nappi, "Dilaton Gravity and No Hair Theorem in Two Dimensions,"
Report No. IASSNA-HEP-92-22 (unpublished); D. A. Lowe, Phys. Rev. D 47, 2446 (1993).

[9] J. Preskill, P. Schwarz, A. Shapere, S. Trivedi, and F. Wilczek, Mod. Phys. Lett. A 6, 2353 (1991).

[10] J. M. McNamara, Proc. R. Soc. London A358, 449 (1978); A364, 121 (1978); Y. Gürsel, V. D. Sandberg, I. D. Novikov, and A. A. Starobinsky, Phys. Rev. D 19, 413 (1979); R. A. Matzner, N. Zamorano, and V. D. Sandberg, ibid. 19, 2821 (1979); S. Chandresekhar and J. B. Hartle, Proc. R. Soc. London A284, 301 (1982); N. Zamorano, Phys. Rev. D 26, 2564 (1982); E. Poisson and W. Israel, Phys. Rev. Lett. 63, 1663 (1989); Phys. Lett. B 233, 74 (1989); Phys. Rev. D 41, 1796 (1990); A. Ori, Phys. Rev. Lett. 67, 789 (1992).

[11] H. Terao, "Two-Dimensional Black Hole Evaporation in Light-Cone Gauge," Kanazawa Report No. 92-19, 1992 (unpublished); X. Shen, Mod. Phys. Lett. A 8, 697 (1993).

[12] T. Banks, A. Dabholkar, M. R. Douglas, and M. O'Loughlin, Phys. Rev. D 45, 367 (1992); J. G. Russo, L. Susskind, and L. Thorlacius, Phys. Lett. B 292, 13 (1992).

[13] D Garfinkle and A. Strominger, Phys. Lett. B 256, 146 (1991). 